\title{
Can flow-volume loops be used to diagnose exercise- induced laryngeal obstructions? A comparison study examining the accuracy and inter-rater agreement of flow- volume loops as a diagnostic tool
}

\author{
*Pernille M Christensen', Niels Maltbæk², Inger M Jørgensen³, Kim G Nielsen ${ }^{4}$ \\ 1 Department of Otorhinolaryngology, Head \& Neck Surgery and Audiology, Rigshospitalet and Faculty of Health and Medical Sciences, \\ University of Copenhagen, Copenhagen, Denmark \\ 2 Pulmonary Department, Roskilde Hospital, University of Copenhagen, Denmark \\ ${ }^{3}$ Paediatric Department, Hillerød Hospital, University of Copenhagen, Denmark \\ ${ }^{4}$ Paediatric Pulmonary Service, Pediatric Department, Rigshospitalet, University of Copenhagen, Denmark
}

Originally received 26th July 2012; resubmitted 21st February 2013; revised 18th April 2013; accepted 24th April 2013; online 18th August 2013

\begin{abstract}
Background: Pre- and post-exercise flow-volume loops are often recommended as an easy non-invasive method for diagnosing or excluding exercise-induced laryngeal obstructions in patients with exercise-related respiratory symptoms. However, at present there is no evidence for this recommendation.

Aims: To compare physician evaluated pre- and post-exercise flow-volume loops and flow data with laryngoscopic findings during exercise.

Methods: Data from 100 consecutive exercise tests with continuous laryngoscopy during the test were analysed. Laryngoscopic images were compared with the corresponding pre- and post-exercise flow-volume loops assessed by four separate physicians and with data from the loops (forced inspiratory flow (FIF) at $25 \%$ vs. FIF at $75 \%$ of forced inspiratory vital capacity (FIVC), forced expiratory flow at $50 \%$ of forced expiratory volume vs. FIF at $50 \%$ of FIVC, and FIVC vs. FIF at $50 \%$ of FIVC).

Results: There was no significant association between the laryngoscopic findings and the flow-volume data. There was no agreement between the four physicians in their assessment of the flow-volume loops (kappa <0.00), and none of the individual physician's assessments were significantly associated with the laryngoscopic findings.

Conclusions: Exercise-induced laryngeal obstructions cannot be diagnosed or excluded by physician evaluated pre- and post-exercise flow-volume loops or flow data alone.

(C) 2013 Primary Care Respiratory Society UK. All rights reserved.

PM Christensen et al. Prim Care Respir J 2013; 22(3): 306-311

http://dx.doi.org/10.4104/pcrj.2013.00067
\end{abstract}

Keywords vocal cord dysfunction, lung function testing, spirometry, exercise, diagnosis

\section{See linked editorial by Morris \& Christopher on pg 267}

\section{Introduction}

In patients with exercise-related respiratory symptoms (ERRS), exercise-induced asthma (EIA) has long been considered the most likely diagnosis. However, studies have shown that other causes for example, exercise-induced laryngeal obstructions (EILOs), chronic obstructive pulmonary disease, obesity and anaemia - should also be considered..$^{1-4}$

While careful history-taking and simple objective examination may help to differentiate between these alternative diagnoses, differentiation between EIA and EILOs requires additional investigations. The symptomatologies are similar ${ }^{1,3,5}$ and, if only one diagnosis is considered, cases with co-morbidity of EIA and EILO may

\footnotetext{
* Corresponding author: Dr Pernille Meliá Christensen, Department of Otorhinolaryngology, Head \& Neck Surgery and Audiology, Rigshospitalet, Faculty of Health and Medical Sciences, University of Copenhagen, Blegdamsvej 9, 2100 Copenhagen, Denmark.

Tel: +4522950504 Fax: +4535452629 E-mail: p.christensen@dadlnet.dk
} 
be missed. In these cases the effect of treatment may well be obscured by the similar symptomatology as only one condition is recognised and treated. The prevalences are similar, making neither diagnosis an exception and co-morbidities not uncommon. ${ }^{1,3,5}$

Currently, the only method for objective verification of ElLOs is laryngoscopic visualisation of the obstruction during exercise. This method gives a detailed description of the type of obstruction. The obstruction is most commonly seen as: (1) a closure/adduction of the vocal folds during inspiration and, to some extent, also during expiration (glottic closure (GC), also known as vocal cord dysfunction or paradoxical vocal fold motion); and/or (2) an inspiratory rotation of the arytenoid region into the airflow (arytenoid rotation, AR) ${ }^{3,5}$ The choice of treatment is dictated by the type of obstruction - for example, speech therapy for GC and surgery has been suggested for $A R$ as well as inspiratory muscle strength training..$^{6 \cdot 8}$ Laryngoscopy is therefore needed to guide treatment; however, it is costly and invasive and can only be done in secondary/tertiary care specialised units.

An alternative to laryngoscopy usable in primary care would be welcomed. Flow-volume loops (FVLs) can be done in most general practices in many countries, so this would be an excellent tool for differentiating between EIA and EILOs. FVLs are often recommended and the method has become accepted in many practices around the world in spite of the fact that the recommendation to use FVLS is based mainly on case reports and a few other inconclusive studies. ${ }^{9-14}$

Owing to the small amount of documentation on the feasibility of FVLs to diagnose ElLOs, the aim of the present study was to assess this feasibility accurately by comparing physician evaluated ${ }^{15}$ pre- and post-exercise FVL and FVL data ${ }^{14,16}$ with laryngeal obstruction measured objectively by laryngoscopy during maximal exercise in a clinically relevant study population.

\section{Methods}

\section{Study design}

The study was designed as a method comparison study comparing the degree of laryngoscopically visualised laryngeal obstruction during maximal exercise with pre- and post-exercise FVL during the same maximal physical stress test.

\section{Study population}

To ensure a clinically relevant study population, participants with varying degrees of exercise-related respiratory symptoms were included. Concurrent asthma was not an exclusion criterion. Data were collected prospectively in the years 2007-2009 from 100 consecutive individuals in whom a maximal physical stress test with continuous laryngoscopy was performed as part of screening for EILOs in the general public in Copenhagen, Denmark aged 14-24 years randomly selected from the Danish Central Population Registry based on their date of $\operatorname{birth}^{5}(n=50)$ and among Danish elite athletes suspected of asthma aged $12-40$ years $(n=50)$. All tests were done at the Pulmonary Department at Bispebjerg Hospital, a tertiary healthcare unit specialising in respiratory medicine. The study was approved by the Scientific Ethics Committee for Region H (KF 01 310187 and H-KF-2007-0033). Informed consent forms were signed by participants (parents/guardians in patients aged $<18$ years) before any study-related examinations were performed.

\section{Measurements}

\section{Maximal physical stress test}

The protocol used has been shown to be able to induce laryngeal obstructions of various degrees, with $74 \%$ of those in whom a moderate/severe obstruction was induced reporting recent ERRS. ${ }^{5}$ The protocol was performed at constant speed with a 3\% increase in slope every $1.5 \mathrm{~min}$ (first $2 \mathrm{~min}$ without inclination) and continued until the test subject felt unable to continue due to exhaustion in spite of encouragement. The speed was individualised with the objective of the subjects reaching exhaustion at 5 min of running $( \pm 1.5 \mathrm{~min})$. The participants were instructed and encouraged to push themselves physically prior to and during the test. All inhaled $1 \mathrm{mg}$ terbutaline (Bricany ${ }^{\circledR}$ Turbuhaler, AstraZeneca, UK) $10 \mathrm{~min}$ prior to the test and were equipped with a heart rate monitor and safety harness.

\section{Laryngoscopy}

The laryngoscopy method has been described in detail elsewhere. ${ }^{17}$ In short, a fibre laryngoscope (Olympus, Shinjuku-ku) is placed through a nostril under local anaesthesia with the tip at the level of the uvula, giving a clear view of the supraglottic and glottic structures of the larynx. The laryngoscope is then fixed with tape at the nose and a hat is worn by the participants so that they can move about freely with the larynx continuously visualised. The laryngoscope stays in place for the duration of the maximal physical stress test.

\section{Flow-volume loops (FVLs)}

A set of three individual FVLs were performed by the subjects immediately before the maximal physical stress test and another set of three loops within $1 \mathrm{~min}$ after exercise (maximal exertion). The same handheld electronic spirometer (EasyOne ${ }^{\circledast}$, ndd Medizintechnik, Zürich, Switzerland) was used for all measurements, and graphs and FVL data were produced which were printed onto paper for each subject. The spirometer was calibrated each day of testing. The same examiner performed all tests.

\section{Evaluation of FVLS}

Four physicians with extensive experience in interpreting FVLs - two adult pulmonologists (raters 1 and 2) and two paediatric pulmonologists (raters 3 and 4) - were asked to diagnose ElLOs based on the printed FVL alone. The raters were blinded to the medical history, name, age, laryngoscopical findings, and the overall number of positive tests. Using forced inspiratory flow at 25\%, 50\% and $75 \%$ of forced inspiratory volume ( $\mathrm{FIF}_{25}, \mathrm{FIF}_{50}$ and $\mathrm{FIF}_{75}$ ), forced inspiratory volume capacity (FIVC) from the loop with the highest FIVC, and forced expiratory flow at 50\% of forced expiratory volume (FEF50) from the loop with the highest forced expiratory volume in one second $\left(\mathrm{FEV}_{1}\right)$ from each set of three $\mathrm{FVLS}$, $\mathrm{FIF}_{25} / \mathrm{FIF}_{75},{ }^{14} \mathrm{FEF}_{50} / \mathrm{FIF}_{50}{ }^{16}$ and $\mathrm{FIVC} / \mathrm{FIF}_{50}$ were calculated.

\section{Evaluation of laryngoscopic recordings}

Each laryngeal recording was evaluated for quality and abnormalities (cysts, asymmetry or similar) by the same experienced examiner. Recordings showing abnormalities or variations of EILOs not categorised as AR or GC or of insufficient quality for evaluation of the degree of obstruction were excluded. The degree of 
obstruction of both AR and GC was evaluated and graded by the same examiner using the following two methods (both methods were used as there is currently no consensus as to which is best):

(1) The CLE-score method ${ }^{18}$ : the degree of $A R$ and GC was evaluated at maximal exertion (within the last 20 seconds of running) and graded 0 for no obstruction, 1 for mild, 2 for moderate, and 3 for maximal obstruction for both AR and GC.

(2) Using EILOMEA software ${ }^{17}$ : a still frame is taken at maximal exertion (within the last 20 seconds of running) showing the maximal inspiratory obstruction and, with the EILOMEA software, the proportion of the potential supraglottic opening above the glottis still open (EILOMEA AR measure) and the angle at the anterior commissure of the vocal folds (EILOMEA GC measure) are determined.

\section{Data analysis}

All statistical analyses were performed using SAS statistical software 9.2 by PMC with support from a statistician in the Biostatistical Department at the University of Copenhagen; $p$ values $<0.05$ were considered significant.

\section{Inter-rater agreement}

The simple kappa value was calculated for inter-rater agreement between raters.

\section{Rater vs. laryngoscopically verified obstruction}

(1) Comparison with total laryngeal obstruction: Using $2 \times 2$ tables calculating $\chi^{2}$ test/Fisher exact test, it was examined whether there was an association between each individual rater's evaluation of the FVL (yes/no) and total laryngeal obstruction defined using the CLE score method (severe/moderate AR and/or GC vs. no moderate/severe AR and GC).

(2) Comparison with degree of AR and GC: One-way ANOVA was used to examine if those evaluated by the individual rater to have EILOs based on the FVL had a higher degree of AR (EILOMEA AR measure) than those not found to have EILOs. This was controlled for sub-population, gender and age, excluding cases with moderate/severe GC (evaluated using the CLE score). This was also repeated looking at the degree of GC (EILOMEA GC measure, excluding cases with moderate/severe AR).

\section{FVL data vs. laryngoscopically verified obstruction}

One-way ANOVA was used to analyse an association between the CLE score and each of the suggested FVL data variables (postexercise FVL data and post-exercise/pre-exercise FVL) controlled for gender, age, sub-population and control of the model. The same was done for the obstruction evaluated using EILOMEA.

\section{Results}

Of the 100 laryngoscopic recordings, two did not have sufficient quality for evaluation of the degree of obstruction and the cases were excluded; no adverse events occurred as a result of performing the tests. Of the remaining 98, 62.2\% were female (age range 12-40 years, median 18 years). All had normal laryngoscopic pre-test findings and the laryngeal obstructions occurring during exercise were either of the AR and/or GC type. Laryngoscopically verified AC and/or GC of moderate/severe degree (ad modum CLE-score) were seen in $62.2 \%$, the angle was measured to mean $43^{\circ}(95 \%$ confidence
Table 1. Overview of kappa values for inter-rater

agreement in evaluating the flow-volume loops for the

diagnosis of exercise-induced laryngeal obstructions

\begin{tabular}{ll} 
& $\begin{array}{l}\text { Kappa values } \\
\text { (95\% confidence interval) }\end{array}$ \\
\hline $\begin{array}{l}\text { Including cases where raters felt } \\
\text { they were unable to evaluate the }\end{array}$ & $-0.07(-0.13$ to 0.00$)$ \\
flow-volume loops ( $3 \times 3$ tables) & \\
\hline $\begin{array}{l}\text { Excluding cases where raters felt } \\
\text { they were unable to evaluate the } \\
\text { flow-volume loops ( } 2 \times 2 \text { tables) }\end{array}$ & $-0.09(-0.39$ to 0.21$)$ \\
\hline
\end{tabular}

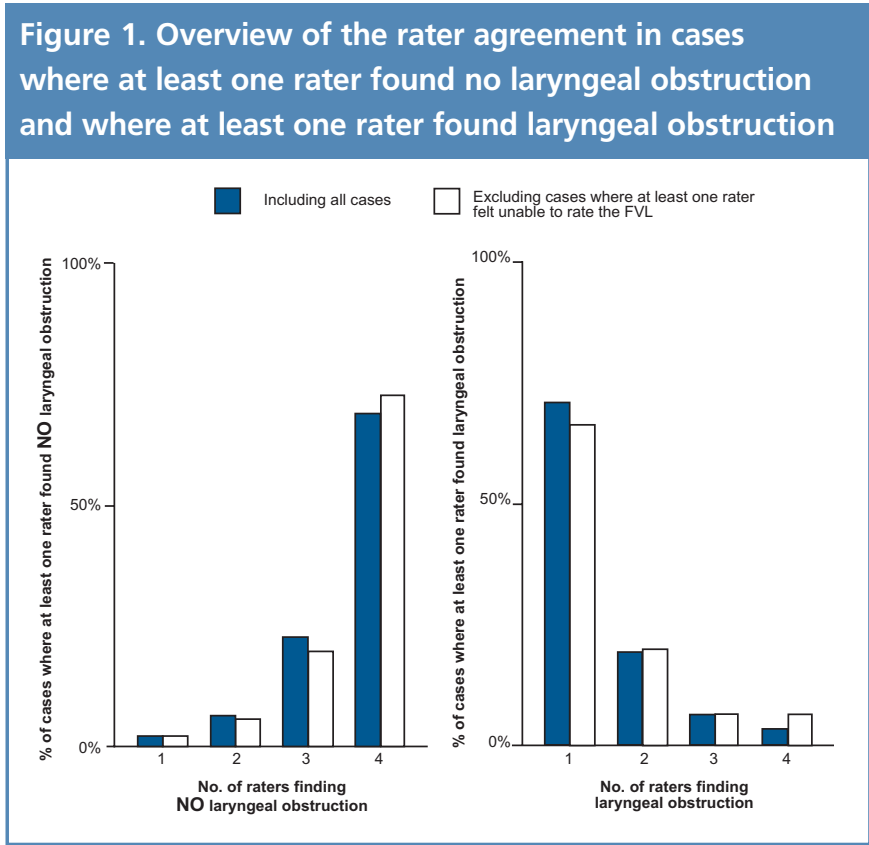

Figure 2. Total laryngeal obstruction (arytenoid rotation and glottic closure) at maximum exertion verified

laryngoscopically and grated ad modum CLE-score against rater evaluation

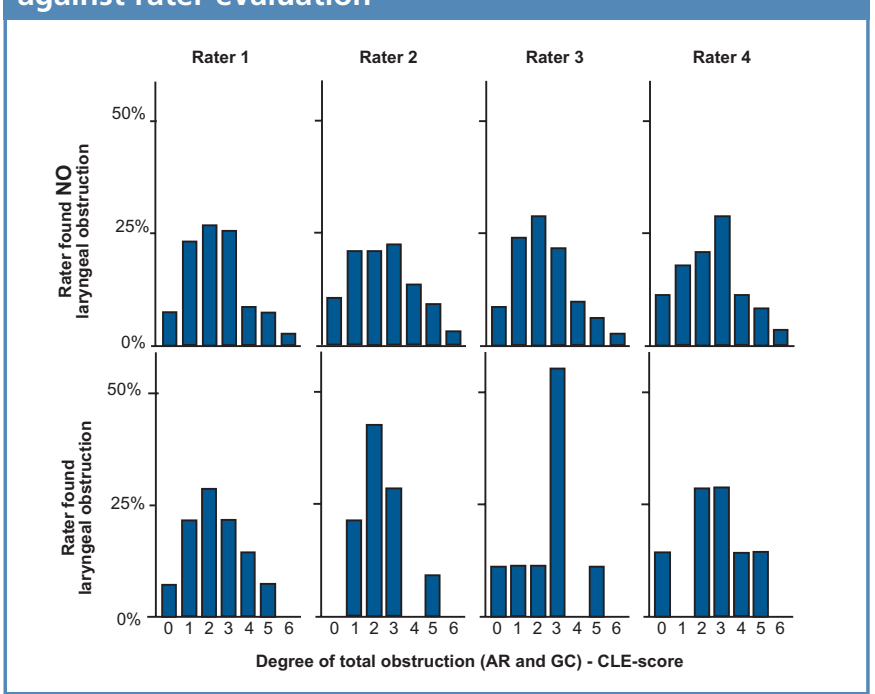




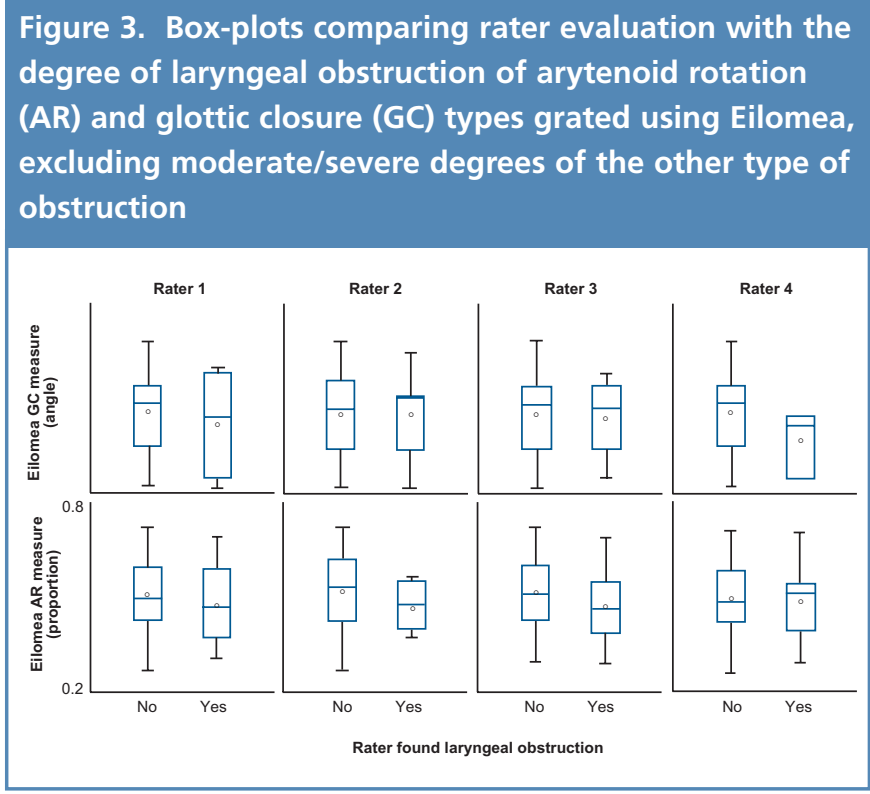

Figure 4. Box-plots comparing degree of total laryngeal obstruction (arytenoid rotation (AR) and glottic closure (GC) grated ad modum Maat) with each of the suggested FVL variables (post-exercise value divided bythe pre-exercise value)

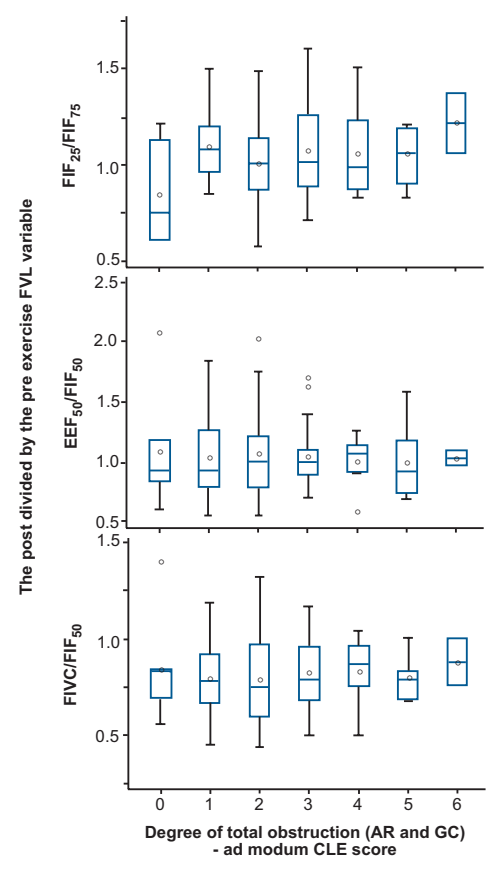

interval $(\mathrm{CI}) 21^{\circ}$ to $\left.64^{\circ}\right)$, and the mean Eilomea AR measure was 0.49 (95\% Cl 0.23 to 0.75 ).

\section{Inter-rater agreement}

There was no agreement between the raters (Table 1, kappa values $<0.00$, defined as no agreement by Landis and $\left.K \mathrm{Koch}^{19}\right)$. At least one rater made a diagnosis of ElLOs in 31 cases, but in only nine of these
Figure 5. Scatter-plots comparing degrees of laryngeal obstruction of arytenoid rotation (AR) and glottic closure (GC) types (using Eilomea, excluding moderate/ severe degrees of the other type of obstruction) with each of the suggested FVL variables (post-exercise value divided by the the pre-exercise value)

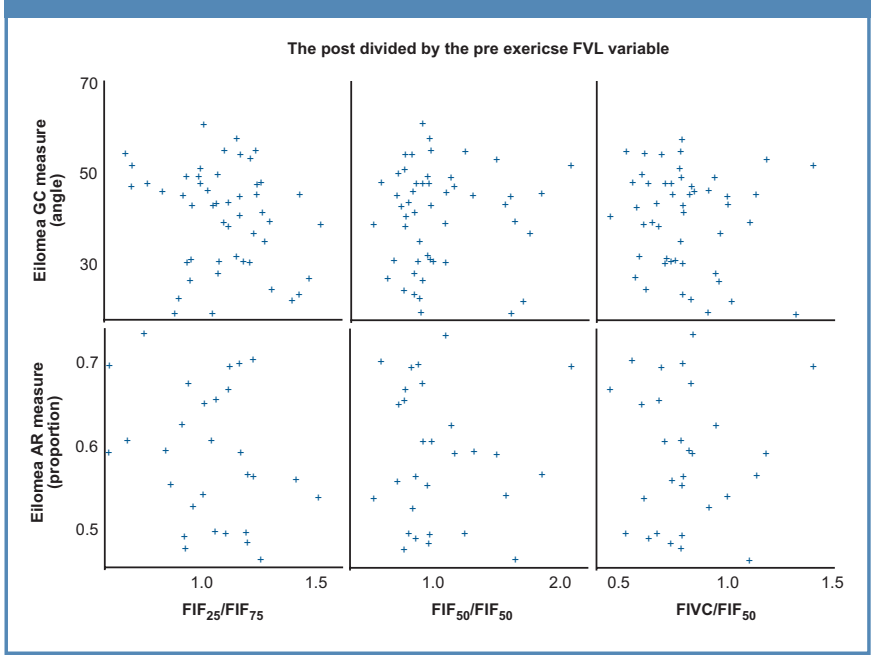

did two or more raters agree and in only one case did all raters agree (Figure 1).

\section{Rater vs. laryngoscopically verified obstruction}

The laryngoscopically observed degree of obstruction of those diagnosed with ElLOs did not differ significantly from the observed degree of obstruction in those diagnosed without ElLOs based on the FVL. This was true for all raters (Figures 2 and 3).

\section{FVL data vs. laryngoscopically verified obstruction}

No associations were found between the objectively measured laryngeal degree of obstruction and any of the FVL data variables (Figures 4 and 5).

\section{Discussion}

\section{Main findings}

This is the first study to assess systematically whether pre- and postexercise FVLs can be used as a surrogate method of objective verification of a laryngeal obstruction induced by exercise. The lack of agreement between the assessments by the four physicians and the finding that none of the raters was able to differentiate between subjects with and without laryngoscopically verified laryngeal obstruction clearly demonstrates that pre- and post-exercise FVLs cannot be used to diagnose or exclude ElLOs. This suggestion was further substantiated by the finding that none of the FVL data variables were associated with the objectively measured degree of laryngeal obstruction. Laryngoscopy therefore remains the best and, at present, the only method for objective verification of ElLOs.

\section{Strengths and limitations of this study}

The conclusions should be seen remembering that all the raters had extensive experience in pulmonology and interpretation of FVLS, including pre- and post-exercise FVLs, but still none of them was able to differentiate between EILOs and no EILOs. Together with the lack 
of association between the FVL data and the degree of obstruction, this makes it very unlikely that other raters or FVL data combinations would be able to produce better results.

All raters complained that the FVLs were difficult to evaluate because they did not know the symptoms of the patients. It is likely that knowing the symptoms could have influenced the assessment. However, the objective of the study was to evaluate the value of FVLS in verifying a laryngeal obstruction and not the symptoms reported by the patients. Blinding the raters was done to ensure just that.

The population was selected to be as representative as possible of the individuals most likely to be examined for ElLOs. Had we chosen only patients with severe obstruction and those without any obstruction, or patients who had been proven not to have any other possible diagnosis that might cause exercise-related respiratory symptoms or influence FVL in any way (e.g. asthma, malnutrition, cardiac disease, other airway obstructions), the results might have been different. However, this is very different from the everyday situation in the clinic and the results could not have been generalised.

Potential explanations for why pre- and post-exercise FVLs did not prove useful as a surrogate diagnostic method include the possibility that the laryngeal obstruction had disappeared before the post-challenge FVL was performed. The post-exercise FVL was taken as quickly as possible after the exercise challenge and it is unlikely that it could have been much earlier. Another reason could be that the participants were too out of breath to produce forced breaths of sufficient power for the laryngeal obstruction to be visualised; however, delaying the measurement of FVL might allow the laryngeal obstructions to subside and the FVL would again prove unusable.

Our experience shows that individuals with EILOs have difficulties in completing a regular exercise test for asthma. It would therefore have been interesting to examine whether the FVL could have been used to diagnose EIA in cases of ElLOs. However, the design used in the study did not allow this to be examined. All participants were given a $\beta_{2}$-agonist (terbutaline) prior to the maximal physical stress test which should have prevented an attack of EIA, and the FVLs were taken within 60 seconds after the end of exercise when EIA is not usually seen on the FVLs. Additionally, the exercise test used did not follow a typical exercise test for EIA. Therefore, any signs of EIA should not have been present on the FVL. There are indications of high co-morbidity of EIA and EILOs. ${ }^{20,21}$ The study would have been stronger had the possibility of using preand post-exercise FVLs to diagnose EIA in cases of EILOs also been examined. However, the lack of this examination does not influence the result of the main findings of the study - namely, that FVLs cannot be used to diagnose ElLOs.

\section{Interpretation of findings in relation to previously published work}

Owing to the nature of EILOs such as the respiratory demands of exercise and the short-lived nature of the attacks after exercise, the requirements of the diagnostic methods differ from other types of obstructions in the larynx. Laryngeal obstructions induced by emotional stressors or other unknown triggers have been described. ${ }^{21}$
It is in these cases that the main challenges for objective verification of the obstruction lie in inducing the attacks in the clinical setting in a standardised manner. However, the attacks may be more prolonged and are not challenged by the respiratory demands of exercise. These types of differences in diagnostic challenges result in different requirements of diagnostic methods and set-ups for various types of laryngeal obstructions. Studies have either to look specifically at one subtype of laryngeal obstruction or ensure evaluation of the results in relation to the nature of the different subtypes in order for the results to be useful in the everyday clinic and to be compared with other studies. At present, no other study of methods available in general practice has been published taking this into account, and the remaining studies have been inconclusive. ${ }^{14}$

Implications for future research, policy and practice The results of this study show that pre- and post-exercise FVLs are unreliable. The method should be abandoned as a diagnostic tool in clinical practice and in research. Patients suspected of EILOs should be referred to specialised clinics with more advanced diagnostic methods - preferably continuous laryngoscopy during an exercise challenge - to confirm or reject the diagnosis. However, this should only be done after other potential causes have been excluded or considered objectively and well-treated despite continued symptoms.

\section{Conclusions}

Exercise-related respiratory symptoms may be caused by a variety of conditions such as asthma and other lung diseases, cardiac disease, obesity, and laryngeal obstructions. ${ }^{1.4}$ The symptoms of EILOs may mimic the symptoms of EIA, and differentiation of these two conditions requires objective measurements. This paper evaluates the feasibility of the commonly recommended and often used FVL to diagnose ElLOs. Pre- and post-exercise FVLs were found to be unreliable for both excluding and verifying the diagnosis of ElLOs. The method should not be used or recommended for use as a method for objective verification of ElLOs.

\section{Handling editor Mohammed Osman Yusuf Statistical review Gopal Netuveli}

Acknowledgements Thanks to Finn Vejlø (Pulmonary Department, Bispebjerg Hospital) for being one of the raters.

Conflicts of interest The authors declare that they have no conflicts of interest in relation to this article.

Contributorship PMC, the guarantor, conceived the idea for the study, wrote the first draft of the protocol, collected and analysed the data, and wrote the first draft of the manuscript. NM, IMJ, and KGN reviewed and refined the protocol, collected the data, and reviewed and refined the draft of the manuscript.

Funding The study was funded by independent grants from Copenhagen University, Anti-Doping Denmark, Team Denmark, and The Danish Agency of Science Technology and Innovation.

\section{References}

1. Morris MJ, Grbach VX, Deal LE, Boyd SYN, Morgan JA, Johnson JE. Evaluation of exertional dyspnea in the active duty patient: the diagnostic approach and the utility of clinical testing. Mil Med 2002;167(4):281-8.

2. Rundell KW, Im J, Mayers LB, Wilber RL, Szmedra L, Schmitz HR. Self-reported symptoms and exercise-induced asthma in the elite athlete. Med Sci Sports Exerc 2001;33(2):208-13

3. Røksund OD, Maat RC, Heimdal JH, Olofsson J, Skadberg BT, Halvorsen T. Exercise induced dyspnea in the young. Larynx as the bottleneck of the airways. Respir Med 2009;103(12):1911-18. http://dx.doi.org/10.1016/j.rmed.2009.05.024 
4. Somerville SM, Rona RJ, Chinn S. Obesity and respiratory symptoms in primary school. Arch Dis Child 1984;59(10):940-4. http://dx.doi.org/10.1136/adc.59.10.940

5. Christensen PM, Thomsen SF, Rasmussen N, Backer V. Exercise-induced laryngeal obstructions: prevalence and symptoms in the general public. Eur Arch Otorhinolaryngol 2011;268(9):1313-19. http://dx.doi.org/10.1007/s00405-011-1612-0

6. Maat RC, Roksund OD, Olofsson J, Halvorsen T, Skadberg BT, Heimdal JH. Surgical treatment of exercise-induced laryngeal dysfunction. Eur Arch Otorhinolaryngol 2007;264(4):401-07. http://dx.doi.org/10.1007/s00405-006-0216-6

7. Echternach M, Delb W, Verse T, Richter B. Does isolated expiratory vocal cord dysfunction exist? Otolaryngol Head Neck Surg 2008;138(6):805-06. http://dx.doi.org/10.1016/j.otohns.2007.12.042

8. Mathers-Schmidt BA, Brilla LR. Inspiratory muscle training in exercise-induced paradoxical vocal fold motion. J Voice 2005;19(4):635-44. http://dx.doi.org/10.1016/j.jvoice.2005.03.005

9. Elshami AA, Tino G. Coexistent asthma and functional upper airway obstruction. Case reports and review of the literature. Chest 1996;110(5):1358-61. http://dx.doi.org/10.1378/chest.110.5.1358

10. Cormier YF, Camus P, Desmeules MJ. Non-organic acute upper airway obstruction: description and a diagnostic approach. Am Rev Respir Dis 1980;121(1):147-50.

11. Wilson JJ, Theis SM, Wilson EM. Evaluation and management of vocal cord dysfunction in the athlete. Curr Sports Med Rep 2009;8(2):65-70.

12. Deckert J, Deckert L. Vocal cord dysfunction. Am Fam Physician 2010;81(2):156-9.
13. Weinberger M, Abu-Hasan M. Pseudo-asthma: when cough, wheezing, and dyspnea are not asthma. Pediatrics 2007;120(4):855-64. http://dx.doi.org/10.1542/peds.2007-0078

14. Watson MA, King CS, Holley AB, Greenburg DL, Mikita JA. Clinical and lung-function variables associated with vocal cord dysfunction. Respir Care 2009;54(4):467-73.

15. Perkins PJ, Morris MJ. Vocal cord dysfunction induced by methacholine challenge testing. Chest 2002;122(6):1988-93. http://dx.doi.org/10.1378/chest.122.6.1988

16. Sterner JB, Morris MJ, Sill JM, Hayes JA. Inspiratory flow-volume curve evaluation for detecting upper airway disease. Respir Care 2009;54(4):461-6.

17. Christensen P, Thomsen SF, Rasmussen N, Backer V. Exercise-induced laryngeal obstructions objectively assessed using ElLOMEA. Eur Arch Otorhinolaryngol 2010;267(3):401-07. http://dx.doi.org/10.1007/s00405-009-1113-6

18. Maat RC, Roksund OD, Halvorsen $T$, et al. Audiovisual assessment of exerciseinduced laryngeal obstruction: reliability and validity of observations. Eur Arch Otorhinolaryngol 2009;266(12):1929-36. http://dx.doi.org/10.1007/s00405-009-1030-8

19. Landis JR, Koch GG. The measurement of observer agreement for categorical data. Biometrics 1977;33(1):159-74. http://dx.doi.org/10.2307/2529310

20. Rundell KW, Spiering BA. Inspiratory stridor in elite athletes. Chest 2003;123(2):46874. http://dx. doi.org/10.1378/chest.123.2.468

21. Christopher KL, Wood RP, Eckert RC, Blager FB, Raney RA, Souhrada J. Vocal-cord dysfunction presenting as asthma. N Engl J Med 1983;308(26):1566-70. http://dx.doi.org/10.1056/NEJM198306303082605

\section{Available online at http://www.thepcrj.org}

